\title{
Summarizing Results for Scaling OMA Mode Shapes by the OMAH Technique
}

\author{
Anders Brandt ${ }^{1}$, Marta Berardengo ${ }^{2}$, Stefano Manzoni ${ }^{3}$, Marcello Vanali ${ }^{2}$, \\ Alfredo $\mathrm{Cigada}^{3}$ \\ ${ }^{1}$ Department of Technology and Innovation, University of Southern Denmark, \\ Campusvej 55, 5230, Odense M, Denmark \\ ${ }^{2}$ Università degli Studi di Parma, Department of Engineering and Architecture, \\ Parco Area delle Scienze, 181/A - 43124 Parma, Italy \\ ${ }^{2}$ Politecnico di Milano, Department of Mechanical Engineering, \\ Via La Masa, 34 - 20156 Milan, Italy
}

\begin{abstract}
Methods for scaling mode shapes determined by operational modal analysis (OMA) have been extensively investigated in the last years. A recent addition to the range of methods for scaling OMA mode shapes is the so-called OMAH technique, which is based on exciting the structure by harmonic forces applied by an actuator. By applying harmonic forces in at least one degree-of-freedom (DOF), and measuring the response in at least one response DOF, while using at least as many frequencies as the number of mode shapes to be scaled, the mode shape scaling (modal mass) of all modes of interest may be determined. In previous publications on the method the authors have proven that the technique is easy and robust to apply to both small scale and large scale structures. Also, it has been shown that the technique is capable of scaling highly coupled modes by using an extended multiple reference formulation. The present paper summarizes the theory of the OMAH method and gives recommendations of how to implement the method for best results. It is pointed out, as has been shown in previous papers, that the accuracy of the mode scaling is increased by using more than one response DOF, and by selecting DOFs with high mode shape coefficients. To determine the harmonic force and responses, it is recommended to use the three-parameter sine fit method. It is shown that by using this method, the measurement time can be kept short by using high sampling frequency and bandpass filtering whereas spectrum based methods require long measurement times. This means that even for structures with low natural frequencies, the extra measurement time for scaling the mode shapes can be kept relatively short.
\end{abstract}

Keywords: Operational Modal Analysis, OMA, Mode Shape Scaling, OMAH, Sine Excitation

\section{INTRODUCTION}

Operational modal analysis (OMA) naturally leads to unscaled mode shapes, since the forces acting on the structure are not measured. It is not uncommon that scaled mode shapes are desired, however. In such cases, several methods exist by which the mode shapes obtained by the OMA parameter extraction may be scaled. Most of the methods developed to scale OMA mode shapes can be divided into the following categories:

1. methods based on several OMA tests, with different mass or stiffness configurations, see for example [1-4];

2. methods based on knowing the mass matrix of the structure, expand the OMA mode shapes to the size of the 
mass matrix, and scale the mode shapes using the weighted mode vector orthogonality property, see [5];

3. methods based on exciting the structure by a known force, and use this force for scaling, usually referred to as OMAX, see for example $[6,7]$.

Of the methods above, the last method has the advantage that it uses an actual measurement of the force, and is thus, in some sense, scaling the modal model to some calibrated force value. On the other hand, it is generally difficult to excite large structures with broadband force. The authors recently suggested to use harmonic forces for the excitation, since this requires less performance of the actuator used [8]. The method, called OMAH, was extended with a global formulation in [9], allowing to use multiple degrees of freedom (DOFs) for force as well as response locations. The global OMAH method is therefore capable of scaling mode shapes also in cases where there is no single DOF to be chosen for excitation of all modes. Furthermore, using several response points for the scaling reduces the variance in the estimates of the modal mass of the structure.

Using harmonic force to scale OMA mode shapes has the advantage that it puts little demand on the actuator, as the actuator only needs to produce a narrowband excitation. Relatively inexpensive actuators can readily be designed for harmonic excitation - even for exciting large structures at low frequencies with relatively high force levels. Furthermore, the estimation of the harmonic signal, hidden in random noise from wind, traffic, and other possible sources, can be achieved under poor signal-to-noise ratios (SNRs), with well-known signal processing methods (mainly the so-called three-parameter sine fit method), see Section 2.2.

\section{THEORY}

The theory of the global formulation of the OMAH method is presented in this section. First, in Section 2.1 by laying out the method for scaling, based on estimates of the frequency response of the structure at a number of frequencies. Secondly, in Section 2.2 the method to accurately determine the harmonic force and responses at a particular frequency, is discussed.

\subsection{OMAH mode shape scaling}

Scaling mode shapes is identical to determining the modal mass of each mode. We start by assuming a frequency response function (FRF) in receptance format (displacement over force) between excitation in DOF $q$ and response in DOF $p$, which can be written as a function of angular frequency, $\omega$, as

$$
H_{p, q}(\mathrm{j} \omega)=\sum_{r=1}^{N} \frac{\psi_{r}^{q} \psi_{r}^{p}}{m_{r}\left(\mathrm{j} \omega-s_{r}\right)\left(\mathrm{j} \omega-s_{r}^{*}\right)}
$$

where $m_{r}$ denotes the modal mass of mode $r$, and * denotes complex conjugate. Moreover, $\psi_{r}^{p}$ and $\psi_{r}^{q}$ are the eigenvector coefficients (from the OMA) for mode $r$ at DOFs $p$ and $q$, respectively. The poles, $s_{r}$, are defined by the undamped natural frequencies (in rad/s), $\omega_{r}$, and the relative damping ratios, $\zeta_{r}$, as

$$
s_{r}=-\zeta_{r} \omega_{r}+\mathrm{j} \omega_{r} \sqrt{1-\zeta_{r}^{2}}
$$

Finally, $\mathrm{j}$ is the imaginary unit.

After OMA parameter extraction all factors on the right-hand side are known, except the modal mass, and scaling the modal model thus requires to determine the modal mass of each mode.

The OMAH method relies on first making an OMA test, whereafter a number of frequency responses, $H_{p, q}(\mathrm{j} \omega)$, are estimated at a number of response DOFs $p=p_{1}, p_{2}, \ldots, p_{m}$ and one or more excitation DOFs $q=q_{1}, q_{2}, \ldots, q_{v}$. Then, an equation system is set up to estimate the modal masses and, potentially, residual terms accounting for out-of-band modes. In the simplest of cases, however, Equation 1 can be used directly employing a single FRF estimate, assuming a single-DOF approximation and no effects of surrounding modes. 
For the general case, we define a global scaling method by first assuming we wish to scale a number, $g$, modes, from mode number $h$ to $h+g-1$, using the set of measured FRFs. We also define constant residual terms $C_{p q}$ (for modes below the modes of interest) and $D_{p q}$ (for modes above the modes of interest) by approximating the FRF by

$$
H_{p, q}(\mathrm{j} \omega) \approx \sum_{r=h}^{h+g-1} \frac{\psi_{r}^{p} \psi_{r}^{q}}{m_{r}\left(\mathrm{j} \omega-s_{r}\right)\left(\mathrm{j} \omega-s_{r}^{*}\right)}+\frac{C_{p q}}{\omega^{2}}+D_{p q} .
$$

Next, we define the FRF column vector $\{H\}_{1}$, containing measured FRFs, by

$$
\begin{aligned}
& \{H\}_{1}=\left[\begin{array}{llllll}
H_{p_{1}, q_{1}}\left(\mathrm{j} \omega_{\mathrm{ex}, 1}\right) & H_{p_{1}, q_{1}}\left(\mathrm{j} \omega_{\mathrm{ex}, 2}\right) & \ldots & H_{p_{2}, q_{1}}\left(\mathrm{j} \omega_{\mathrm{ex}, 1}\right) & H_{p_{2}, q_{1}}\left(\mathrm{j} \omega_{\mathrm{ex}, 2}\right) & \ldots
\end{array}\right. \\
& \begin{array}{llllll}
H_{p_{m}, q_{1}}\left(\mathrm{j} \omega_{\mathrm{ex}, 1}\right) & H_{p_{m}, q_{1}}\left(\mathrm{j} \omega_{\mathrm{ex}, 2}\right) & \ldots & H_{p_{1}, q_{2}}\left(\mathrm{j} \omega_{\mathrm{ex}, 1}\right) & H_{p_{1}, q_{2}}\left(\mathrm{j} \omega_{\mathrm{ex}, 2}\right) & \ldots
\end{array} \\
& \left.H_{p_{m}, q_{v}}\left(\mathrm{j} \omega_{\mathrm{ex}, 1}\right) \quad H_{p_{m}, q_{v}}\left(\mathrm{j} \omega_{\mathrm{ex}, 2}\right) \quad \ldots\right]^{\mathrm{T}}
\end{aligned}
$$

where the superscript []$^{\mathrm{T}}$ denotes vector transpose. Furthermore, we define the column vector $\{x\}_{1}$ with the unknown modal masses and residual terms, by

$$
\begin{aligned}
& \{x\}_{1}=\left[\frac{1}{m_{h}} \quad \frac{1}{m_{h+g-1}} \ldots\right. \\
& C_{p_{1} q_{1}} \quad D_{p_{1} q_{1}} \quad C_{p_{2} q_{1}} \quad D_{p_{2} q_{1}} \ldots \\
& \begin{array}{llllll}
C_{p_{m} q_{1}} & D_{p_{m} q_{1}} & C_{p_{1} q_{2}} & D_{p_{1} q_{2}} & \ldots
\end{array} \\
& \left.C_{p_{m} q_{v}} \quad D_{p_{m} q_{v}}\right]^{\mathrm{T}} \text {. }
\end{aligned}
$$

We now introduce the function $\Gamma\left(p, q, r, \omega_{\mathrm{ex}}\right)$, defined by:

$$
\Gamma\left(p, q, r, \omega_{\mathrm{ex}}\right)=\frac{\psi_{r}^{p} \psi_{r}^{q}}{\left(\mathrm{j} \omega_{\mathrm{ex}}-s_{r}\right)\left(\mathrm{j} \omega_{\mathrm{ex}}-s_{r}^{*}\right)}
$$

at one of the experimental frequencies $\omega_{\mathrm{ex}}$ where the $\mathrm{FRF}$ is measured. Finally we build a matrix $[A]_{1}$, defined by

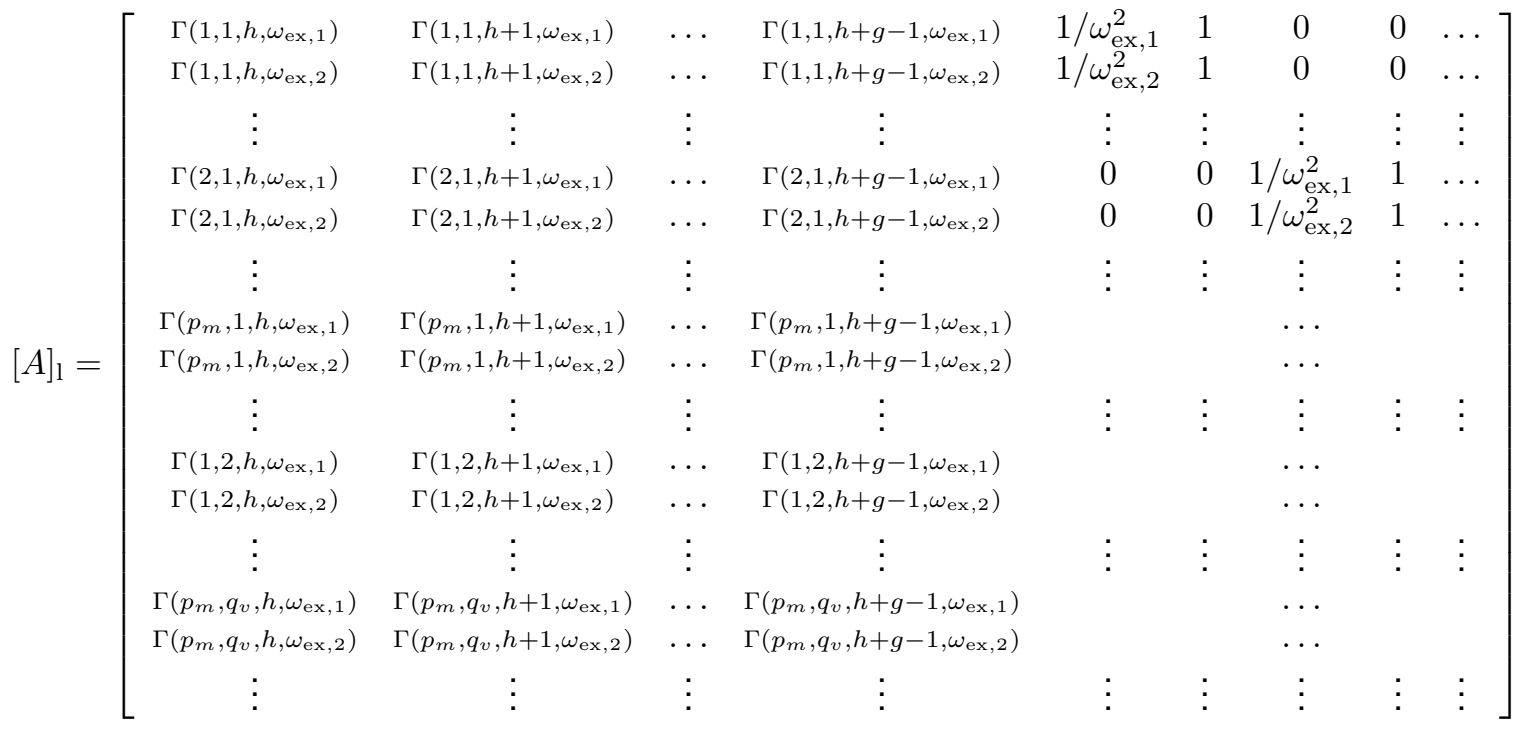

at various frequencies $\omega_{\mathrm{ex}, \mathrm{k}}$ for some integers $k$.

Using the vectors and matrices thus defined, we can form an equation system

$$
[H]_{1}=[A]_{1}\{x\}_{1}
$$


which can be solved for the vector $\{x\}_{1}$ by a least squares solution or a pseudo inverse.

It is important to note that the frequencies in each line of the matrix $[A]_{1}$, although for simplicity denoted $\omega_{\mathrm{ex}, 1}, \omega_{\mathrm{ex}, 2}, \ldots$, may actually be arbitrary frequencies, albeit in most cases it will be practical to calculate many of the FRFs from simultaneous measurements of several responses.

Next, it is important to consider which requirements apply in order to make the matrix $[A]_{1}$ well conditioned. First, we need to ensure that we have more rows in the equation system than the number of unknowns (i.e. modal masses plus residual terms). If we have measured $M$ unique $\operatorname{FRF}$ locations $(p, q)$, then it is sufficient that the number of lines in the equation system, $L>g+2 M$. In most cases this will be fulfilled without any extra thought, if the recommendations in Section 2.3 are followed.

Next, we note that each function $\Gamma\left(p, q, r, \omega_{\mathrm{ex}, \mathrm{k}}\right)$ in the matrix $[A]_{1}$, belongs to the FRF value $H_{p, q}\left(\mathrm{j} \omega_{\mathrm{ex}, \mathrm{k}}\right)$ due to mode $r$, at frequency $\omega_{\mathrm{ex}, \mathrm{k}}$. The natural way to ensure that $[A]_{1}$ is well conditioned, is to excite the structure at frequencies close to the eigenfrequencies and including all the modes to be scaled. Also it should be ensured that the excitation DOF in each case, is at a point of the structure where the mode in question is well excited (i.e. has a large mode shape coefficient). This will ensure that each line in $[A]_{1}$ has at least one large function $\Gamma\left(p, q, r, \omega_{\mathrm{ex}, \mathrm{k}}\right)$.

\subsection{Estimating harmonics in noise}

One of the strengths with the OMAH method, is that harmonics hidden in noise can be accurately and efficiently estimated even in cases with very low SNRs. The method therefore does not rely on large excitation forces. Furthermore, as will be shown in the present section, the measurement time does not have to be very long even in cases with poor SNRs.

The method we recommend for estimating the complex amplitudes of the force and response signals $F_{q}\left(\mathrm{j} \omega_{\mathrm{ex}}\right)$ and $U_{p}\left(\mathrm{j} \omega_{\mathrm{ex}}\right)$ is the so-called three-parameter sine fit method [10]. The name implies that the DC value as well as two Fourier coefficients $a$ and $b$ are unknown, whereas in our case the DC does not apply since we remove the mean of the signals prior to estimating the harmonics. Regardless, the method is usually referred to as the three-parameter sine fit.

For simplicity, in the following we assume that we are exciting the structure at a frequency $\omega$ (corresponding to one of the frequencies previously denoted $\omega_{\mathrm{ex}, \mathrm{k}}$. We furthermore assume we have measured $N$ samples of the signal $y(t)$ at $y(n)=y(n \cdot \Delta t)\left(y(t)\right.$ being a force or a response signal) using a sampling frequency $f_{s}=1 / \Delta t$. The measured signal will then conform to the model

$$
y(t)=a \cos (\omega t)+b \sin (\omega t)+e(t)
$$

where the Fourier coefficients $a$ and $b$ are the desired unknowns, and $e(t)$ contains the random part of the signal, due to wind, traffic, and other random contributions, and possibly higher (and undesired) harmonics of the excitation frequency. To calculate the complex FRF values, we calculate the amplitude and phase of $y(n)$ as $\sqrt{a^{2}+b^{2}}$. $\exp (\mathrm{j} \arctan (b / a))$.

Based on the measurements of $y(n)$, we can define the matrix $B$

$$
[B]=\left[\begin{array}{cc}
\cos (\omega \cdot 0 \Delta t) & \sin (\omega \cdot 0 \Delta t) \\
\cos (\omega \cdot 1 \Delta t) & \sin (\omega \cdot 1 \Delta t) \\
\cos (\omega \cdot 2 \Delta t) & \sin (\omega \cdot 2 \Delta t) \\
\vdots & \\
\cos (\omega \cdot(N-1) \Delta t) & \sin (\omega \cdot(N-1) \Delta t)
\end{array}\right]
$$


and the unknown coefficient vector $\{x\}=\left[\begin{array}{ll}a & b\end{array}\right]^{\mathrm{T}}$, and the measurement vector

$$
\{y\}=\left[\begin{array}{c}
y(0) \\
y(1) \\
y(2) \\
\vdots \\
y(N-1)
\end{array}\right] .
$$

The model in Equation (9), can now be written as:

$$
[B]\{x\}=\{y\}+\{e\}
$$

which we can solve for the estimate $\hat{x}$ of $x$ in Equation (12), by a least squares solution. Furthermore, having solved for the estimate $\hat{x}$, the remaining signal $\hat{e}$ may be calculated by

$$
\{\hat{e}\}=\{y\}-\{B\}\{\hat{x}\} .
$$

after which we can estimate the variance of the remaining signal $\hat{e}$ by

$$
\sigma_{\hat{e}}^{2}=\frac{\{\hat{e}\}^{T}\{\hat{e}\}}{N} .
$$

Since the power of the unknown harmonic is $\left(\hat{a}^{2}+\hat{b}^{2}\right) / 2$ the SNR can be calculated as

$$
\mathrm{SNR}=\frac{\hat{a}^{2}+\hat{b}^{2}}{2 \sigma_{\hat{e}}^{2}}=\frac{\hat{x}^{\mathrm{T}} \hat{x}}{2 \sigma_{\hat{e}}^{2}} .
$$

When the coefficients in $\{\hat{x}\}$ are computed, the absolute value of $\hat{F}_{q}$ that we need for the FRF estimates, may be computed by

$$
\left|\hat{F}_{q}(\mathrm{j} \omega)\right|=\sqrt{\hat{x}^{\mathrm{T}} \hat{x}}
$$

In [10] it is shown that the mean square errror (MSE) of $\left|\hat{F}_{q}(\mathrm{j} \omega)\right|$ in Equation (16) is approximately

$$
\operatorname{MSE}\left[\hat{F}_{q}\right] \approx \frac{2 \sigma_{\hat{e}}^{2}}{N}
$$

which is a conservative error.

The result in Equation (17) is a very important result. It shows, that at any SNR (i.e. any level of variance of the random part of the reponse, independent of the harmonic amplitude), the relative error in the amplitude can be made arbitrarily small, by increasing the number of samples, $N$. Furthermore, it is important to realize that this number of samples can be recorded in a short time, by increasing the sampling frequency without increasing the bandwidth of the measurement system, since this will not affect the SNR, but will result in more samples in a given time. In most modern measurement systems, this will not be allowed because the cutoff frequency is chosen as a factor of the selected sampling frequency. But a higher performance can be achieved by sampling at a high frequency, and then bandpass filtering the data after the data acquisition is finished (which will improve the SNR as well as create more samples).

If we want to have a particular maximum uncertainty on the estimate of the FRF value, we can allow half that uncertainty on each of the force and response values. Furthermore, since the MSE includes both random and bias errors, a conservative measure may be to use three times the square root of the MSE as the maximum deviation from the measured values. Say, for example, that we may allow $5 \%$ inaccuracy on the FRF estimate. For the force (and similar for the response) measurements, we then need to assure that,

$$
100 \frac{3 \cdot \sqrt{\operatorname{MSE}\left[\hat{F}_{q}\right]}}{\left|\hat{F}_{q}\right|} \leq 2.5
$$




\subsection{Guidelines for applying OMAH}

Using the results in Sections 2.1 and 2.2, we will now present some guide lines for the design of a test for scaling mode shapes from an OMA test. The following procedure can thus be used.

1. Complete the OMA measurements. This is usually done by measuring batches of channels, keeping some references. Keep all the sensors in place after the last batch measurement. If all DOFs can be measured simultaneously, the better.

2. Complete the OMA parameter estimation, resulting in poles and unscaled mode shapes.

3. Study the mode shapes, and choose a DOF with large mode shape coefficients for some (if possible most) modes. Attach the actuator in this DOF. (In many cases where the mode shapes are, at least approximately, known beforehand, this actuator position can be determined prior to performing the OMA test.)

4. Add an accelerometer on the mass of the actuator, for measuring the force. If there is no available channel, replace one of the response channels with the accelerometer for measuring the force.

5. Investigate a proper force amplitude, by measuring an arbitrary amount of time, and estimate the signals $F_{q}(t)$ and $U_{p}(t)$, where $p$ denotes all the DOFs in the last measured batch. Furthermore, $U_{q}$ must be included in the set of $U_{p}$. This requires estimating the MSE using Equation (17), and to calculate an appropriate relative error. If necessary, increase the sampling frequency and follow up by lowpass filtering the signals prior to estimating the amplitudes and phases, to ensure the measurement time is kept appropriately short. This step may also involve changing the moving mass of the actuator, to produce an appropriate force.

6. For each frequency, $\omega_{\mathrm{ex}, \mathrm{k}}$, compute the amplitudes and phases of the force and all responses, and compute the FRF values $H_{p, q}\left(\mathrm{j} \omega_{\text {ex }, \mathrm{k}}\right)$ and store these FRF values.

7. Make consecutive measurements of frequencies near all modes which are well excited by the DOF $q$ where the actuator is located. This means that data for creating a relatively large number of rows for the matrix $[A]_{1}$ in Equation (7) will be acquired.

8. For the mode or modes not well excited by the first chosen excitation DOF, move the actuator to a DOF where one or more of these modes will be well excited. Excite the mode or modes near their natural frequency (frequencies), measure the force and all response channels, produce the new FRF values, and store these. In most cases, each of these modes needs to be excited only at a single frequency near the natural frequency of the mode in question in order to be able to solve Equation (8). However, exciting more frequencies, as long as they are close to a mode that is well excited by the DOF $q$, can result in higher accuracy of the estimated modal masses. Repeat this step until all modes have been well excited at least at one frequency.

9. Now build the measurement vector $\{H\}_{1}$ and the matrix $[A]_{1}$ by Equations (4) and (7), respectively, and solve for the unknown modal masses and residual terms by solving Equation (8).

\section{DISCUSSION}

To see how an OMAH test may be conducted, we assume we would scale the first 8 vertical modes of the Little Belt Bridge, see [11]. The information about the modes is found in Table 1. We also assume that we have an eightchannel measurement system, and that we use seven accelerometers for the response measurements for scaling, including the reference (in the same DOF as the force). The final channel is used to measure the acceleration of the moving mass of the actuator, so we can compute the excitation force. Using the information in Table 1 and information about the mode shapes (that are essentially the shapes of a pinned narrow plate), we define three excitation points, in order to be able to excite all modes well:

1. vertically on one side, at $1 / 4$ from one end, to excite modes $1,2,5,7,8$

2. vertically on one side, at $1 / 3$ from one end, to excite modes 4 , and 6

3. vertically on one side, at $1 / 2$ from one end, to excite mode 3 


\begin{tabular}{rrrl}
$\begin{array}{r}\text { Mode } \\
\#\end{array}$ & $\begin{array}{r}\text { Frequency } \\
{[\mathrm{Hz}]}\end{array}$ & $\begin{array}{r}\text { Damping } \\
{[\%]}\end{array}$ & Description \\
\hline & & & \\
1 & 0.155 & 1.49 & First vertical bending \\
2 & 0.171 & 11.41 & Second vertical bending \\
3 & 0.258 & 0.69 & Third vertical bending \\
4 & 0.402 & 0.75 & Fourth vertical bending \\
5 & 0.524 & 0.65 & First torsion \\
6 & 0.572 & 0.45 & Fifth vertical bending \\
7 & 0.769 & 0.71 & Sixth bending \\
8 & 0.807 & 0.72 & Second torsion \\
\hline
\end{tabular}

Table 1: First 8 modes of the Little Belt Bridge, used as an example for a mode shape scaling case

Note that these may not be optimal points, but are given as an example.

We thus start the test by positioning the actuator in position 1, and set the frequency close to $0.155 \mathrm{~Hz}$. Since mode 1 has a damping value of $1.5 \%$, we need to wait approximately 500 seconds for the structure to reach its steady-state condition. After this time, we acquire a number of samples, for example corresponding to five periods of the harmonic, i.e. $32.3 \mathrm{~s}$. After calculating the complex sines of the force and the 7 responses, we check that Equation (18) is fulfilled, and if not, we may set the sampling frequency higher, acquire data again, and BP filter data to a narrow bandwidth around the frequency of interest, and then recompute the complex amplitudes and using them to produce seven estimates of the FRFs.

After this, we tune the frequencies of modes 2, 5, 7, and 8, in turn, and for each of them wait for steady state, acquire data, and check the accuracy. When the accuracy is adequate, we compute the complex amplitudes, and then the FRF values. Once this is accomplished, the shaker is moved to position 2 , the frequency tuned to the frequencies of modes 4 and 6 and the procedure is repeated. Finally, the same is done for position 3 for mode 3 .

When all this is done, we have acquired 7 FRFs times 5 frequencies from position 1, 7 FRFs times 2 frequencies from position 2 and finally 7 FRFs times 1 frequency for position 3 . This thus produces 56 rows in the matrix $[A]_{1}$ in Equation (7). The number of unknowns we have are eight modal masses, plus 2 residuals times 7 response DOFs times 3 excitation DOFs, which equals 50 unknowns. We can thus solve the equation system. It would be advantageous, however, if time allows, to measure a few more frequencies to obtain a more overdetermined system of equations. This could easily be achieved by adding those frequencies, in each position of the actuator, for which there is a reasonably large mode shape coefficient in the forcing DOF.

So, how long would this whole test take? In Table 2 we present all measurements with the settling time and the time taken for measuring five periods of each frequency of excitation. We have defined steady-state conditions as the time when the RMS of the response from one block of data containing five periods to the next five periods, does not change more than $1 \%$. As can be seen in the table, the total time of data acquisition is approximately 45 minutes, of which the major time is spent waiting for the system to reach steady-state conditions. The time to move the actuator is not taken into account in this example. Also note, that the measurement time is independent of the SNR used. So the actuator can, for example, be set to generate $10 \%$ of the RMS of the random response. This example shows that even on a relatively low-frequency structure like this, the OMAH method does not require very long measurement times.

A further advantage with the OMAH technique is that, once the modes are scaled, the accuracy of the scaling can be investigated. This was demonstrated in the previous papers presenting the method, see [8,9]. It is done by comparing the measured harmonic response amplitudes in the response DOFs, with the computed responses using the synthesized FRF (using the scaled modal model) times the excitation force.

Finally, it should also be mentioned that an advantage of the OMAH method is that the response of the structure to changes in the amplitude of the force can easily be included in the measurements. Thus, the linearity of the 


\begin{tabular}{ccrrrr}
$\begin{array}{c}\text { Meas. } \\
\#\end{array}$ & $\begin{array}{c}\text { Exc. } \\
\text { pos. }\end{array}$ & $\begin{array}{r}\text { Mode } \\
\#\end{array}$ & $\begin{array}{r}\text { Frequency } \\
{[\mathrm{Hz}]}\end{array}$ & $\begin{array}{r}\text { Settling } \\
\text { Time [s] }\end{array}$ & $\begin{array}{r}\text { Measurement } \\
\text { Time [s] }\end{array}$ \\
\hline & & & & & \\
2 & 1 & 1 & 0.155 & 500 & 32.3 \\
2 & 1 & 2 & 0.171 & 75 & 29.2 \\
3 & 1 & 5 & 0.524 & 300 & 9.5 \\
4 & 1 & 7 & 0.769 & 220 & 6.5 \\
5 & 1 & 8 & 0.807 & 200 & 6.2 \\
6 & 2 & 4 & 0.402 & 300 & 12.4 \\
7 & 2 & 6 & 0.572 & 400 & 8.7 \\
8 & 3 & 3 & 0.258 & 600 & 19.4 \\
\hline
\end{tabular}

Table 2: Values of settling times (time until the response is steady-state, defined by less than $1 \%$ change in RMS level from 5 periods to next 5 periods), and measurement times ( 5 periods) for the entire test. See text for details. The total time in columns 5 and 6 adds up to $2595 \mathrm{~s}$, or approx. 45 minutes

structure may be investigated. This is not easily done with most other methods for scaling OMA mode shapes.

\section{CONCLUSIONS}

In this paper we have described the theory of global OMAH scaling of mode shapes, using harmonic excitation. The method depends on exciting the structure in one or more DOFs, and,although not strictly necessary, we recommend exciting at frequencies close to the eigenfrequencies of the structure. The least squares global solution method described in the paper can handle structures with closely coupled modes. Furthermore, it gives modal masses for all the modes taken into account as well as residual terms for all the pairs of measuring and forcing points.

It has been shown that the technique offers several attractive properties:

- It puts low demands on the actuator, as the force level can be low relative to ambient response.

- The accuracy of the method may be increased by using more response measurements.

- The method can handle closely coupled modes.

- The sine hidden in noise can be accurately determined without needing long measurement times.

- The method allows to investigate the accuracy of the mode shape scaling, by comparing the measured responses with those from synthesized frequency response multiplied by the harmonic force applied.

- The method allows to easily investigate the linearity, by observing the response for several different force levels.

An example of how to scale the first eight modes of a bridge with eigenfrequencies from approx. 0.15 to $0.8 \mathrm{~Hz}$ was shown to require approximately 45 minutes of total measurement time, exciting the structure in three DOFs, at a total of eight frequencies.

\section{REFERENCES}

[1] E. Parloo, P. Verboven, P. Guillaume, and M. Van Overmeire, "Sensitivity-Based Operational Mode Shape Normalisation," Mechanical Systems and Signal Processing, vol. 16, no. 5, pp. 757-767, 2002.

[2] D. Bernal, "Modal Scaling from Known Mass Perturbations," Journal of engineering mechanics, vol. 130, no. 9, pp. 1083-1088, 2004. 
[3] G. Coppotelli, "On the estimate of the FRFs from operational data," Mechanical Systems and Signal Processing, vol. 23, pp. 288-299, Feb. 2009.

[4] M. L. Aenlle, P. Fernandez, R. Brincker, and A. Fernandez-Canteli, "Scaling-factor estimation using an optimized mass-change strategy," Mechanical Systems and Signal Processing, vol. 24, no. 5, pp. 1260-1273, 2010.

[5] M. L. Aenlle and R. Brincker, "Modal scaling in operational modal analysis using a finite element model," International Journal of Mechanical Sciences, vol. 76, pp. 86-101, Nov. 2013.

[6] E. Reynders, D. Degrauwe, G. De Roeck, F. Magalhães, and E. Caetano, "Combined Experimental-Operational Modal Testing of Footbridges," Journal of Engineering Mechanics, vol. 136, no. 6, pp. 687-696, 2010.

[7] J. Cara, "Computing the modal mass from the state space model in combined experimental operational modal analysis," Journal of Sound and Vibration, vol. 370, pp. 94-110, May 2016.

[8] A. Brandt, M. Berardengo, S. Manzoni, and A. Cigada, "Scaling of mode shapes from operational modal analysis using harmonic forces," Journal of Sound and Vibration, vol. 407, pp. 128-143, Oct. 2017.

[9] A. Brandt, M. Berardengo, S. Manzoni, M. Vanali, and A. Cigada, "Global scaling of operational modal analysis modes with the omah method," Mechanical Systems and Signal Processing, in press.

[10] P. Händel, "Amplitude estimation using IEEE-STD-1057 three-parameter sine wave fit: Statistical distribution, bias and variance," Measurement, vol. 43, pp. 766-770, July 2010.

[11] S. S. Christensen, M. S. Andersen, and A. Brandt, "Dynamic characterization of the little belt suspension bridge by operational modal analysis," in Proc. 36th International Modal Analysis Conference (IMAC), Orlando, FL, 2018. 